\title{
Heat shock protein expression in canine osteosarcoma
}

\author{
Mariarita Romanucci • Giuliana D'Amato - Daniela Malatesta • Laura Bongiovanni • \\ Chiara Palmieri • Andrea Ciccarelli • Paolo Buracco • Emanuela Morello • \\ Lorella Maniscalco • Raffaella De Maria • Marina Martano • Leonardo Della Salda
}

Received: 22 July 2011 /Revised: 13 September 2011 /Accepted: 19 September 2011 / Published online: 21 October 2011

(C) Cell Stress Society International 2011

\begin{abstract}
Abnormal levels of heat shock proteins have been observed in a number of human neoplasms and demonstrate prognostic, predictive and therapeutic implications. Since osteosarcoma (OSA) in dogs provides an important model for the same disease in humans, the aim of this study was to evaluate the immunohistochemical expression of Hsp27, Hsp72, Hsp73 and Hsp90 in 18 samples of canine appendicular OSA, in relation to histological grade and overall survival (OS), in order to investigate their potential prognostic, predictive and/or therapeutic value. A semiquantitative method was used for the analysis of the results. Hsp27, Hsp73 and Hsp90 showed a variably intense, cytoplasmic and nuclear immunoreactivity that was not associated with histological type or grade. On the other hand, a high percentage of
\end{abstract}

This work was presented at the VIII ${ }^{\circ}$ meeting of the Italian Association of Veterinary Pathology (AIPVet), 15-17 June 2011, Padova, Italy. It has also been presented as a poster at the $29^{\text {th }}$ meeting of the European Society of Veterinary Pathology (ESVP), 7-10 September 2011, Uppsala, Sweden.

M. Romanucci · G. D'Amato · D. Malatesta $\cdot$ L. Bongiovanni $\cdot$

C. Palmieri $\cdot$ L. Della Salda $(\triangle)$

Department of Comparative Biomedical Sciences,

Faculty of Veterinary Medicine, University of Teramo,

Teramo, Italy

e-mail: ldellasalda@unite.it

A. Ciccarelli

Department of Communication Sciences, University of Teramo,

Teramo, Italy

P. Buracco • E. Morello • L. Maniscalco • R. De Maria •

M. Martano

Department of Animal Pathology, Faculty of Veterinary Medicine, University of Turin,

Turin, Italy
Hsp72 immunostaining was significantly associated with grade III $(P<0.01)$ and a lack of immunolabelling was significantly correlated to a longer OS $(P=0.006)$. Neoplastic emboli were occasionally positive for Hsp27, faintly immunoreactive for Hsp72 and intensely immunolabelled by Hsp73 and Hsp90. In conclusion, absence of Hsp72 immunosignal appears to be associated with a favourable prognosis whilst the widespread Hsp90 immunoreactivity detected in all tumour cases as well as in neoplastic emboli, suggests this protein could be targeted in the therapy of canine OSA, and likewise in its human counterpart.

Keywords Heat shock proteins · Osteosarcoma $\cdot$ Animal model $\cdot$ Dog

\section{Introduction}

Heat shock proteins (HSPs), also known as stress proteins, are one of the most evolutionarily conserved classes of molecule and play a fundamental role in the maintenance of cellular homeostasis, under both physiological and stress conditions, acting as 'molecular chaperones' (Calderwood et al. 2006). HSPs can be classified into several families, named according to their approximate molecular weight, expressed in kilodaltons, even though new guidelines for the nomenclature of the human HSP families have been proposed (Kampinga et al. 2009). A growing body of evidence suggests that HSPs are implicated in all phases of cancer from proliferation, impaired apoptosis and sustained angiogenesis to invasion and metastasis (Calderwood et al. 2006). Since abnormal HSP levels have been observed in a wide range of human tumours, a number of studies have been carried out in order to determine whether these proteins could be used as 
diagnostic, prognostic and/or predictive markers or represent new molecular targets for cancer therapy (Ciocca and Calderwood 2005; Karapanagiotou et al. 2009). In particular, several studies have been performed to investigate the expression and prognostic significance of HSPs in human osteosarcoma (OSA; Trieb et al. 1998; 2000; Uozaki et al. 2000; Ozger et al. 2009; Moon et al. 2010). In addition, during the last decade, researches have been focused on the potential usefulness of Hsp90 inhibitors as novel treatment approaches of cancer (Porter et al. 2010), including OSA (Gazitt et al. 2009), particularly in the context of childhood sarcomas (Bagatell et al. 2005; 2007). HSPs expression has also been demonstrated in preliminary studies carried out on canine neoplasms (Kumaraguruparan et al. 2005; Romanucci et al. 2005; 2006; Carrasco et al. 2011), which show an abnormal expression pattern similar to that observed in human neoplasms (Kumaraguruparan et al. 2005; Romanucci et al. 2005; 2006). These parallel findings underline the relevance of studying the multiple roles of HSPs in carcinogenesis in animal models as an additional source of information for clinical cancer research. In fact spontaneously occurring tumours in dogs provide an important model for human cancer biology studies and therapeutic strategies, and OSA is considered one of the canine malignancies of most interest as it shows striking similarities in tumour biology and behaviour to its human counterpart (Mueller et al. 2007). However, to the best of our knowledge, no data are available concerning HSP expression in canine OSA tissues.

The aim of this study was to evaluate the immunohistochemical expression and localization of different HSPs in canine OSA and to establish whether this expression could be related to histological tumour grade and overall survival (OS). This study also aimed to shed further light on the role of these proteins in the pathogenesis of canine OSA and therefore their potential prognostic, predictive and/or therapeutic value.

\section{Methods}

Histological examination

The study was carried out on surgical samples from 18 canine appendicular OSA. All tumour cases were supplied by the Department of Animal Pathology, Faculty of Veterinary Medicine, University of Turin (Italy). Samples were fixed in 10\% neutral-buffered formalin and embedded in paraffin wax. Histological diagnosis was established using haematoxylin and eosin stained slides, according to WHO guidelines (Slayter et al. 1994), while histological grade was determined according to the system proposed by Kirpensteijn et al. (2002).
Immunohistochemistry

Formalin-fixed, paraffin-embedded samples were also processed using an immunohistochemical technique with a variety of specific antibodies (Abs) namely, anti-Hsp27 (1:300, rabbit polyclonal, StressGen, Victoria, BC, Canada), anti-Hsp72 (1:50, C92F3A-5, mouse monoclonal, StressGen), anti-Hsp73 (1:100, 1B5, rat monoclonal, StressGen), anti-Hsp90 (1:600, AC88, mouse monoclonal, StressGen) Abs.

Deparaffined and rehydrated sections were incubated in 3\% hydrogen peroxide in absolute methanol for $45 \mathrm{~min}$ to inhibit endogenous peroxidase activity, then rinsed in $0.05 \mathrm{M}$ Tris-buffered saline (TBS), $\mathrm{pH}$ 7.6, for $5 \mathrm{~min}$. Antigen retrieval was performed by heat-treatment in citrate buffer at $\mathrm{pH} 6$ in a microwave oven for $5 \mathrm{~min}$ (three cycles). To reduce non-specific binding, slides were incubated in normal goat serum (Biospa, Milan, Italy) for $10 \mathrm{~min}$ at room temperature before overnight incubation with primary $\mathrm{Ab}$ in a humidified chamber at $4^{\circ} \mathrm{C}$. After rinsing with TBS, immune complexes were treated at room temperature for 10 min with secondary biotinylated Goat anti-mouse/rabbit (ready-to-use, Biospa) or Rabbit anti-Rat (1:100, Dako, Copenhagen, Denmark) Abs and subsequently detected using streptavidin peroxidase (Biospa). Tissue peroxidase activity was detected by $5 \mathrm{~min}$ application of $0.1 \%$ hydrogen peroxide in 3,3'-diaminobenzidine solution (D5905, Sigma-Aldrich, St Louis, Missouri) and followed by counterstaining with Mayer's Haematoxylin for $1 \mathrm{~min}$ before rinsing, dehydrating and mounting.

A negative control was performed in all instances by omitting the primary $\mathrm{Ab}$ and incubating tissue sections with TBS and/or with an irrelevant $\mathrm{Ab}$ directed against an unrelated antigen such as rabbit anti-human von Willebrand factor polyclonal $\mathrm{Ab}$ or mouse anti-human desmin monoclonal Ab (Dako, Glostrup, Denmark).

\section{Clinical follow-up}

Survival data concerning the dogs were supplied by the Department of Animal Pathology, Faculty of Veterinary Medicine, University of Turin (Italy). All the dogs included in the present study had no evidence of metastasis at presentation and a histologically confirmed appendicular OSA. In all cases work-up included history, physical exam, complete blood count, serum biochemical profile, urinalysis and abdominal ultrasound. Limb (LL and AP views) and chest (LL, right and left, and DV views) radiographic evaluation was performed to examine respectively features and extention of the tumour and presence of lung metastasis; computed tomography was performed in case of suspicious lung radiographs. 
Regional lymph nodes were aspirated and cytologically examined when enlarged. Initial diagnosis was attempted by fine needle aspiration and cytology but, for a more specific identification of the tumour type, a peroperative biopsy was obtained in all cases using a Jamshidi needle and submitted to histopathology. For a more precise tumour characterization, histopathology was again performed on the entire tumoural specimen postoperatively. All dogs included in the present study were surgically treated (amputation or limb sparing) before receiving adjuvant chemotherapy using doxorubicin $\left(30 \mathrm{mg} / \mathrm{m}^{2}\right.$, four to five administrations, 21 days apart) or cisplatin (70 $\mathrm{mg} / \mathrm{m}^{2}$, four to five administrations, 21 days apart) as a single agent or a combination of cisplatin and doxorubicin (four cycles, 21 days apart, each cycle consisting of cisplatin $50 \mathrm{mg} / \mathrm{m}^{2}$ at day 1 and doxorubicin $15 \mathrm{mg} / \mathrm{m}^{2}$ at day 2). Local tumour control (surgery) was followed by chemotherapy administration since it has been proved that adjuvant chemotherapy can improve survival in dogs with appendicular OSA, even though no protocol has been demonstrated to be superior (Dernell et al. 2007). Therefore, the protocol was decided based on both literature data and owner's decision (Straw et al. 1991; Berg et al. 1995; Chun et al. 2005). Besides, histopathological tumour grade never influenced the choice of the chemotherapeutic protocol adopted. Canine patients were clinically and radiographically examined every 3 months during the first year after the conclusion of chemotherapy and then every 6 months for a minimum of 2 years. For the animals that died of tumour-related causes within the 2 year period, OS was considered the days between surgery and death, whilst for dogs that survived $>2$ years, OS was the number of days from surgery to the last clinical examination. OS data in relation to chemotherapeutic protocol, histological type and grade are shown in Table 1.

\section{Statistical analysis}

A semiquantitative immunohistochemical assessment (absent, no positive cells; low, $>0$ to $<10 \%$ positive cells; moderate, $\geq 10$ to $<50 \%$ positive cells; high, $\geq 50 \%$ positive cells) was made and Fisher's exact test was used to compare HSP expression with histological grades. Kaplan-Meier analysis was used to estimate survival, and the significances of the differences were determined by the log-rank test. For this purpose, the cases were grouped according to absence/presence of immunostaining or to the expression score as follows: $<10 \%$ positive cells (absent+low semiquantitative evaluation) versus $\geq 10 \%$ positive cells (moderate + high semiquantitative evaluation) or $<50 \%$ positive cells (absent/low + moderate
Table 1 Tumour grade, histological type, post-surgical treatment and overall survival of the cases included in the present study

\begin{tabular}{lllr}
\hline & Histological type & Post-surgical treatment & Overall survival (days) \\
\hline Grade I & & & 1,033 \\
No. 1 & Fibroblastic osteosarcoma & Doxorubicin & 449 \\
No. 2 & Productive osteoblastic osteosarcoma & Cisplatin/doxorubicin & 1,250 \\
No. 3 & Fibroblastic osteosarcoma & Cisplatin/doxorubicin & 1,089 \\
No. 4 & Nonproductive osteoblastic osteosarcoma & Cisplatin/doxorubicin & \\
Grade II & & & 168 \\
No. 5 & Chondroblastic osteosarcoma & Cisplatin/doxorubicin & 553 \\
No. 6 & Mixed osteosarcoma & Cisplatin/doxorubicin & 503 \\
No. 7 & Mixed osteosarcoma & Doxorubicin & 715 \\
No. 8 & Poorly differentiated osteosarcoma & Doxorubicin & 496 \\
No. 9 & Fibroblastic osteosarcoma & Cisplatin/doxorubicin & 2,209 \\
No. 10 & Chondroblastic osteosarcoma & Cisplatin/doxorubicin & 261 \\
No. 11 & Teleangectatic osteosarcoma & Cisplatin/doxorubicin & 391 \\
No. 12 & Productive osteoblastic osteosarcoma & Cisplatin/doxorubicin & \\
Grade III & & & 169 \\
No. 13 & Productive osteoblastic osteosarcoma & Cisplatin/doxorubicin & 242 \\
No. 14 & Productive osteoblastic osteosarcoma & Cisplatin/doxorubicin & 335 \\
No. 15 & Nonproductive osteoblastic osteosarcoma & Doxorubicin & 211 \\
No. 16 & Giant cell type osteosarcoma & Cisplatin & 200 \\
No. 17 & Productive osteoblastic osteosarcoma & Cisplatin/doxorubicin & \\
No. 18 & Giant cell type osteosarcoma & Cisplatin/doxorubicin & \\
\hline & & & \\
\hline
\end{tabular}


semiquantitative evaluation) versus $\geq 50 \%$ positive cells (high semiquantitative evaluation). Analyses were performed using the SPSS statistical software, and the conventional 5\% level was used to define statistical significance.

\section{Results}

\section{HSP expression in canine OSA}

Hsp27 appeared to be weakly to moderately expressed in most tumour cases with a predominantly cytoplasmic localization, rarely nuclear (Fig. 1); only one case was negative. A strong cytoplasmic immunolabelling of multinucleated giant cells was found independently of the immunoreactivity of the surrounding tumour cells (Fig. 1, inset). Neoplastic emboli were not consistently positive, although they reflected the expression observed in the primary tumour.

Hsp72 immunolabelling differed among the various samples examined in intensity of immunosignal and percentage of positive cells; the highest signal was usually noted in tumour cells surrounding necrotic areas. Both cytoplasmic and nuclear localizations were detected, although nuclear signal was more frequent (Fig. 2). Multinucleated giant cells appeared negative whilst lymphatic emboli were negative or only faintly positive (Fig. 2, inset), independently of the immunoreactivity of the primary tumour. Four cases were negative for Hsp72.

Hsp73, as well as Hsp90, exhibited a widespread, moderate to high, cytoplasmic and nuclear positivity in all tumours examined (Figs. 3-4). An intense and diffuse

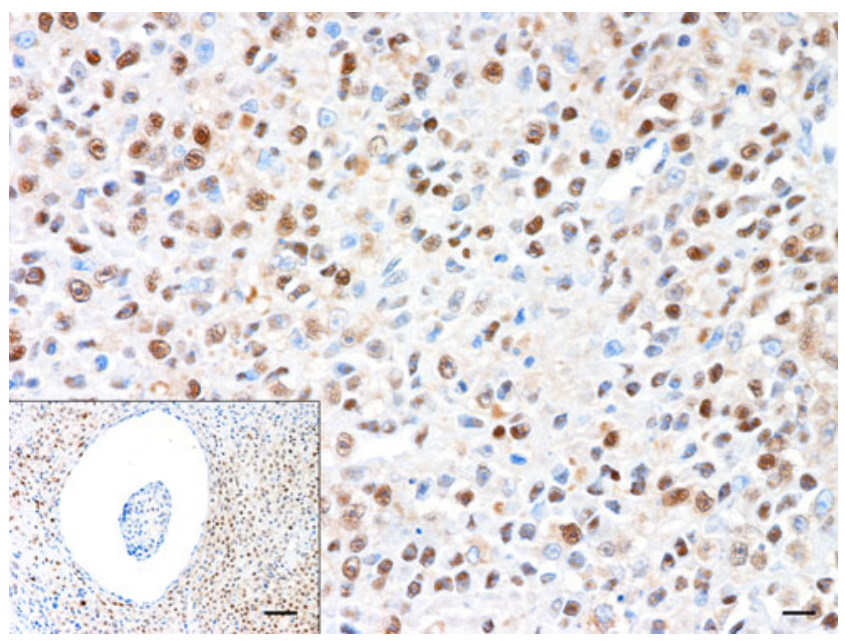

Fig. 2 Nonproductive osteoblastic osteosarcoma: intense Hsp72 immunosignal predominantly located in the nucleus of tumour cells $($ bar $=10 \mu \mathrm{m})$. Inset absence of immunolabelling in a lymphatic embolus $($ bar $=60 \mu \mathrm{m})$

reactivity in lymphatic emboli was also observed (Fig. 4, inset). Multinucleated giant cells showed a characteristic multifocal intense nuclear immunosignal for Hsp73 (Fig. 3, inset) but were inconsistently positive for Hsp90, with either cytoplasmic or nuclear localization.

No significant differences in HSP immunoexpression were found in relation to the histological type of OSA.

\section{Relationship between HSP expression and tumour grades}

The expression of Hsp27, Hsp73 and Hsp90 did not appear to be related to histological grade. High levels of Hsp72

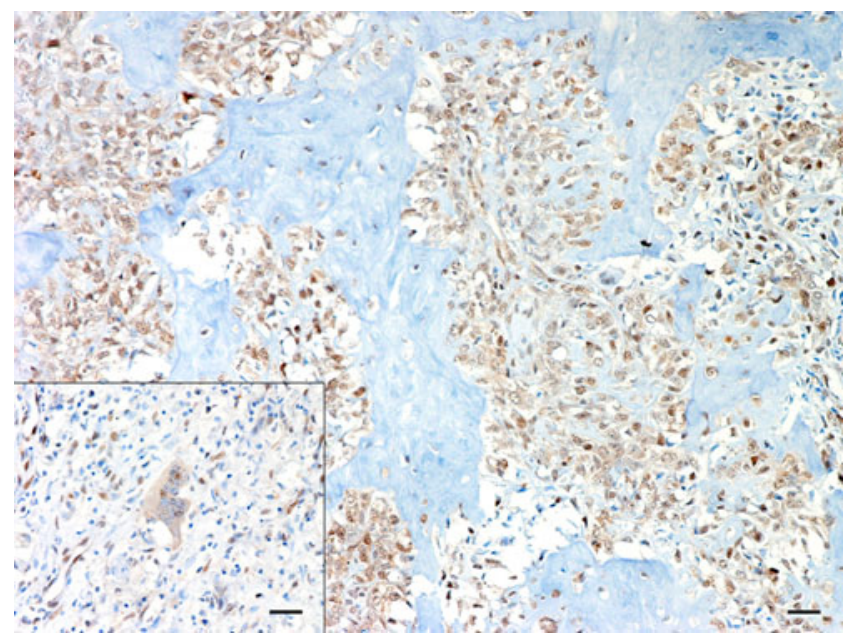

Fig. 3 Productive osteoblastic osteosarcoma: intense, diffuse, cytoplasmic and nuclear Hsp73 immunoreactivity (bar $=22 \mu \mathrm{m})$. Inset scattered, strong nuclear positivities associated with low cytoplasmic immunosignal in a multinucleated giant cell $($ bar $=30 \mu \mathrm{m})$ 


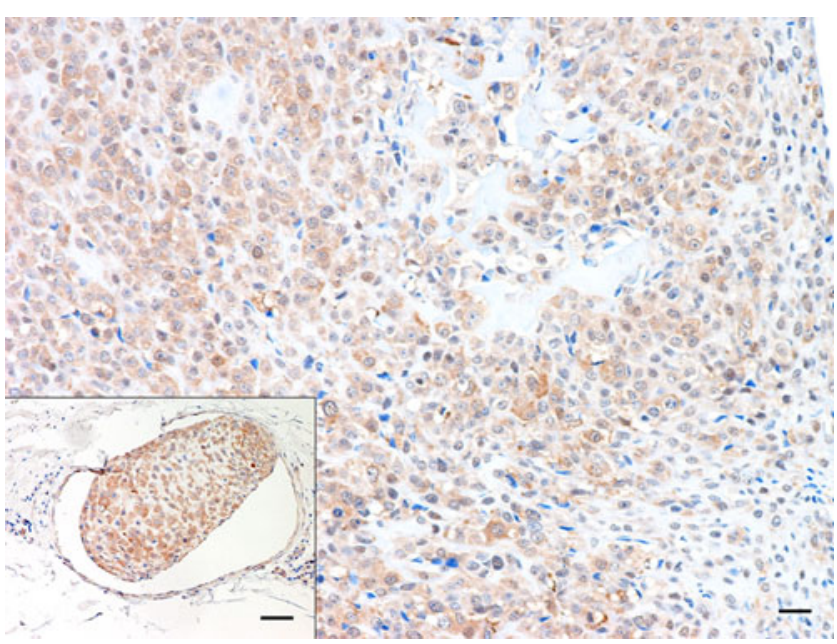

Fig. 4 Productive osteoblastic osteosarcoma: intense and diffuse cytoplasmic Hsp90 immunolabelling in primary tumour cells as well as in a lymphatic embolus (inset) (bar=22 $\mu \mathrm{m}$; inset bar $=60 \mu \mathrm{m})$

immunostaining, on the other hand, were significantly associated to high-grade (grade III) OSA $(P=0.009)$. Expression scores of the different HSPs under study in relation to histological grades are illustrated in Fig. 5.

\section{Relationship between HSP expression and OS}

Survival analysis revealed that absence of Hsp72 immunosignal was significantly associated to a longer post-surgical OS $(P=0.006)$ when compared with cases exhibiting Hsp72

\section{Hsp27}

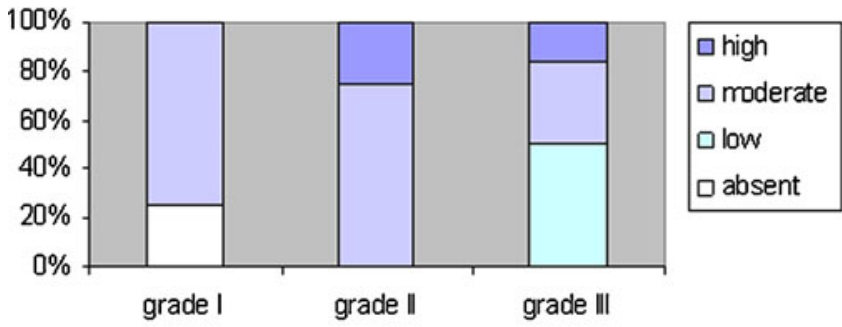

\section{$\mathrm{Hsp} 73$}

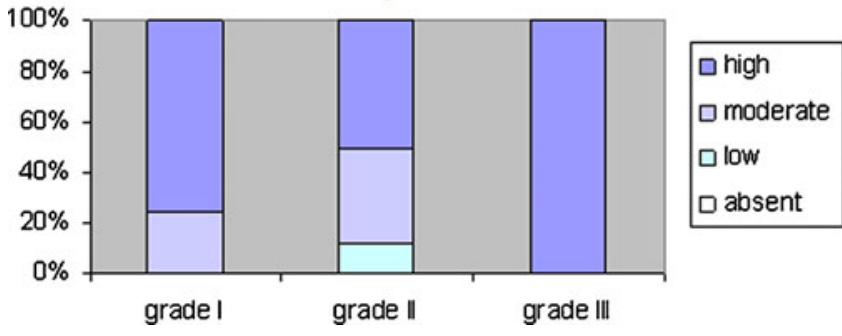

Fig. 5 The figure shows the percentage of expression of each Hsp (absent, low, moderate, high) in the different histological grades of canine osteosarcoma. Hsp27, Hsp73 and Hsp90 expression did not immunoreactivity. However, a significant association with OS was not found when Hsp72 expression scores were taken into consideration, although its lower expression levels $(<10 \%)$ indicated a trend toward a prolonged OS $(P=$ 0.058; Fig. 6). No significant relationship was found between Hsp27, Hsp73 and Hsp90, and OS.

\section{Discussion}

This study demonstrates the immunohistochemical expression of several members of the major HSP families in canine OSA. Similar studies have been carried out in human OSA (Trieb et al. 1998; Uozaki et al. 2000; Ozger et al. 2009; Moon et al. 2010). However, results published in literature are conflicting: Hsp27, Hsp60 and Hsp70 overexpression have been related to poor prognosis by some studies (Uozaki et al. 2000) whilst others have failed to demonstrate any significant influence on the survival rate in human OSA (Ozger et al. 2009; Moon et al. 2010). The results of the present study do not show a significant relationship between the expression scores of the different HSPs evaluated and OS, although absence of Hsp72 immunosignal appeared to be related to a longer postsurgical OS $(P=0.006)$, with also a tendency toward a prolonged OS for its lower expression levels $(P=0.058)$, and a significant association was found between high Hsp72 immunodetection and high-grade OSA. Given the poor prognostic significance of high histological grade in
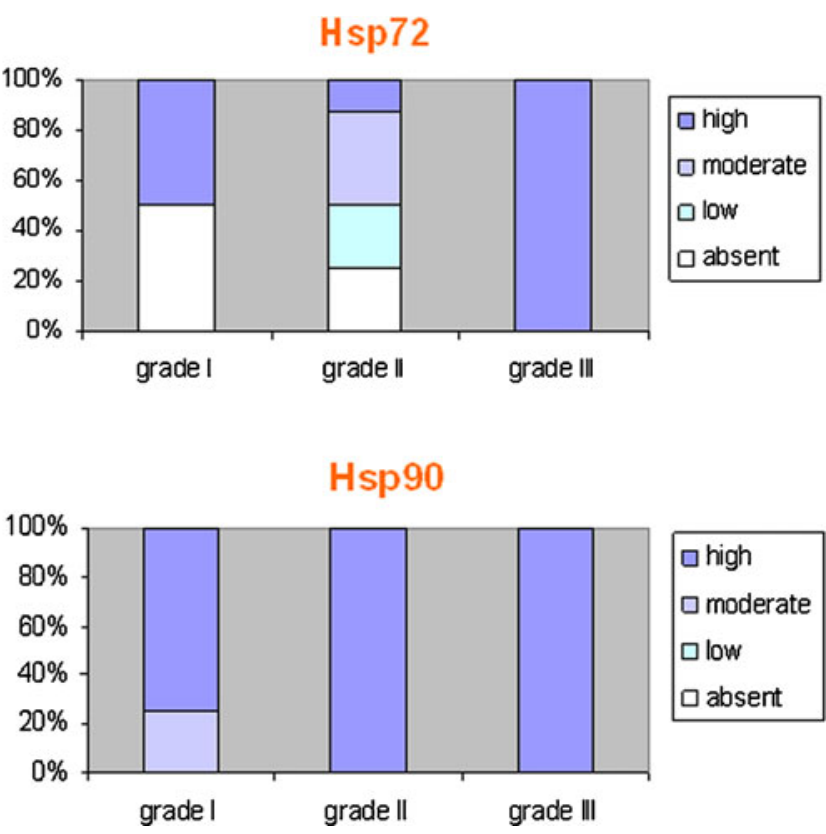

appear to be related to histological grade whereas high percentage of Hsp72 immunostaining was significantly associated with high-grade (grade III) osteosarcoma $(P=0.009)$ 
a

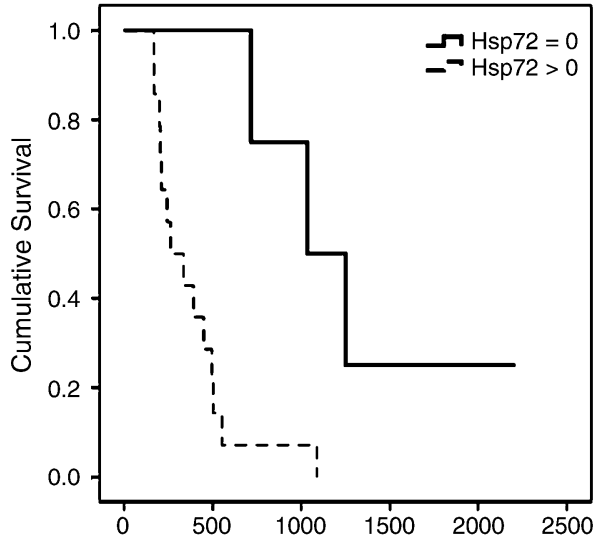

b

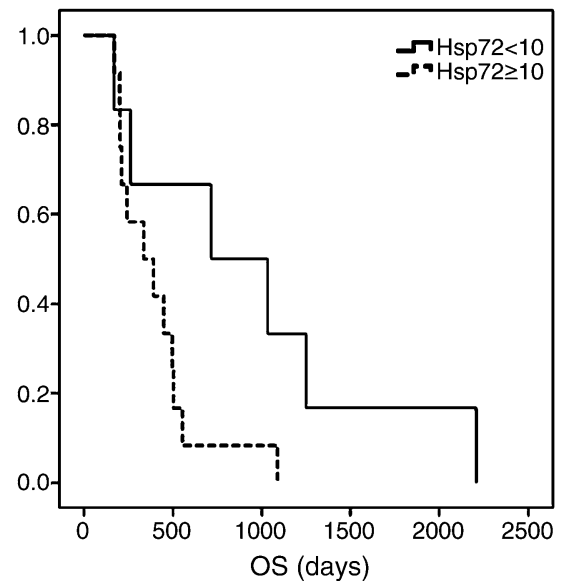

C

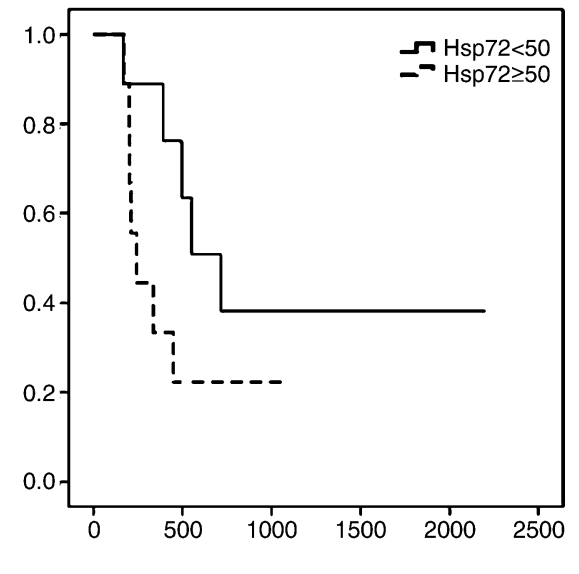

Fig. 6 Kaplan-Meier plots showing influence on survival of Hsp72 immunoexpression: a Absence of Hsp72 immunodetection appeared to be significantly associated to a longer post-surgical OS $(P=0.006)$; b-c Hsp72 expression scores did not show a significant relation to OS $(P=0.058-P=0.112)$

expression on the response to these chemotherapeutic agents. On the other hand, Hsp27 has been hypothesized to be involved in drug resistance of OSA cells to zoledronic acid (Morii et al. 2010), whose adjuvant potential has been highlighted in several studies carried out in animal models of OSA, although its efficacy against pulmonary metastasis remains controversial (Ory et al. 2005; Dass and Choong 2007; Labrinidis et al. 2010).

Both Hsp73 and Hsp90 were the most abundantly expressed proteins in canine OSA. These findings appear to be in line with the participation of the constitutive member of the HSP70 family in the regulation of protein folding, assembly and degradation, as well as in the protection of cellular proteins from the damage caused by cellular stress like hypoxia, thus possibly conferring proliferative advantage on tumour cells. Our results concerning Hsp90 correspond to its role as a specialized molecular chaperone, responsible for folding numerous oncogenic proteins and thus leading to an 'addiction' to this Hsp by cancer cells (Trepel et al. 2010). Information regarding its expression in human OSA tissues is limited (Uozaki et al. 2000; Ozger et al. 2009), although several studies have highlighted the possible effectiveness of Hsp90-binding agents, particularly 17-allylamino-17-demethoxygeldanamicin (17-AAG), in the targeted therapy of this kind of tumour, especially in the context of childhood sarcomas (Bagatell et al. 2005; 2007; Gazitt et al. 2009). In addition, McCleese et al. (2009) have recently demonstrated the selective cytotoxicity of the novel Hsp90 inhibitor STA-1474 for human and canine OSA cell lines. However, to the best of our knowledge, HSP expression in canine OSA samples has not been investigated. The results of this study suggest the involvement of Hsp90 in the pathogenesis of canine OSA although no association seems to exist 
between its expression and tumour prognosis, similarly to humans (Uozaki et al. 2000; Ozger et al. 2009). Lastly, the widespread expression of this protein observed both in primary tumours and in neoplastic emboli strongly highlights the possibility of using a canine model for further testing of Hsp90-targeted cancer therapy.

Acknowledgements We thank Tania Bastow for the linguistic review of the manuscript. This work was completed with grant support from the Italian Ministry of University and Scientific Research, Rome, Italy.

\section{References}

Bagatell R, Beliakoff J, David CL, Marron MT, Whitesell L (2005) Hsp90 inhibitors deplete key anti-apoptotic proteins in pediatric solid tumor cells and demonstrate synergistic anticancer activity with cisplatin. Int J Cancer 113:179-188

Bagatell R, Gore L, Egorin MJ, Ho R, Heller G, Boucher N, Zuhowski EG, Whitlock JA, Hunger SP, Narendran A, Katzenstein HM, Arceci RJ, Boklan J, Herzog CE, Whitesell L, Ivy SP, Trippett TM (2007) Phase I pharmacokinetic and pharmacodynamic study of 17 $\mathrm{N}$-allylamino-17-demethoxygeldanamycin in pediatric patients with recurrent or refractory solid tumors: a pediatric oncology experimental therapeutics investigators consortium study. Clin Cancer Res 15:1783-1788

Berg J, Weinstein MJ, Springfield DS, Rand WM (1995) Results of surgery and doxorubicin chemotherapy in dogs with osteosarcoma. J Am Vet Med Assoc 206:1555-1560

Calderwood SK, Khaleque MA, Sawyer DB, Ciocca DR (2006) Heat shock proteins in cancer: chaperones of tumorigenesis. Trends Biochem Sci 31:164-172

Cardoso F, Di Leo A, Larsimont D, Gancberg D, Rouas G, Dolci S, Ferriera F, Paesmans M, Piccart M (2001) Evaluation of HER2, p53, bcl-2, topoisomerase II-alpha, heat shock proteins 27 and 70 in primary breast cancer and metastatic ipsilateral lymph nodes. Ann Oncol 12:615-620

Carrasco V, Canfrán S, Rodríguez-Franco F, Benito A, Sáinz A, Rodríguez-Bertos A (2011) Canine gastric carcinoma: immunohistochemical expression of cell cycle proteins (p53, p21, and p16) and heat shock proteins (Hsp27 and Hsp70). Vet Pathol 48:322-329

Chun R, Garrett LD, Henry C, Wall M, Smith A, Azene NM (2005) Toxicity and efficacy of cisplatin and doxorubicin combination chemotherapy for the treatment of canine osteosarcoma. J Am Anim Hosp Assoc 41:382-387

Ciocca DR, Calderwood SK (2005) Heat shock proteins in cancer: diagnostic, prognostic, predictive, and treatment implications. Cell Stress Chaperones 10:86-103

Dass CR, Choong PFM (2007) Zoledronic acid inhibits osteosarcoma growth in an orthotopic model. Mol Cancer Ther 6:3263-3270

Dernell WS, Ehrhart NP, Straw RC, Vail DM (2007) Tumors of the skeletal system. In: Withrow SJ, Vail DM (eds) Withrow \& MacEwen's Small animal clinical oncology, 4th edn. Elsevier, St Louis, MO, pp 540-582

Garrido C, Ottavi P, Fromentin A, Hammann A, Arrigo AP, Chauffert B, Mehlen P (1997) Hsp27 as a mediator of confluencedependent resistance to cell death induced by anticancer drugs. Cancer Res 57:2661-2667

Gazitt Y, Kolaparthi V, Moncada K, Thomas C, Freeman J (2009) Targeted therapy of human osteosarcoma with 17AAG or rapamycin: characterization of induced apoptosis and inhibition of mTOR and Akt/MAPK/Wnt pathways. Int J Oncol 34:551561

Kampinga HH, Hageman J, Vos MJ, Kubota H, Tanguay RM, Bruford EA, Cheetham ME, Chen B, Hightower LE (2009) Guidelines for the nomenclature of the human heat shock proteins. Cell Stress Chaperones 14:105-111

Karapanagiotou EM, Syrigos K, Saif MW (2009) Heat shock protein inhibitors and vaccines as new agents in cancer treatment. Expert Opin Investig Drugs 18:161-174

Kirpensteijn J, Kik M, Rutteman GR, Teske E (2002) Prognostic significance of a new histological grading system for canine osteosarcoma. Vet Pathol 39:240-246

Kumaraguruparan R, Karunagaran D, Balachandran C, Manohar BM, Nagini S (2005) Of humans and canines: a comparative evaluation of heat shock and apoptosis-associated proteins in mammary tumors. Clin Chim Acta 365:168-176

Labrinidis A, Hay S, Liapis V, Findlay DM, Evdokiou A (2010) Zoledronic acid protects against osteosarcoma-induced bone destruction but lacks efficacy against pulmonary metastases in a syngeneic rat model. Int J Cancer 127:345-354

McCleese JK, Bear MD, Fossey SL, Mihalek RM, Foley KP, Ying W, Barsoum J, London CA (2009) The novel HSP90 inhibitor STA1474 exhibits biologic activity against osteosarcoma cell lines. Int J Cancer 125:2792-2801

Moon A, Bacchini P, Bertoni F, Olvi LG, Santini-Araujo E, Kim YW, Park YK (2010) Expression of heat shock proteins in osteosarcomas. Pathology 42:421-425

Morii T, Ohtsuka K, Ohnishi H, Mochizuki K, Satomi K (2010) Inhibition of heat-shock protein 27 expression eliminates drug resistance of osteosarcoma to zoledronic acid. Anticancer Res 30:3565-3571

Mueller F, Fuchs B, Kaser-Hotz B (2007) Comparative biology of human and canine osteosarcoma. Anticancer Res 27:155-164

Ory B, Heymann M, Kamijo A, Gouin F, Heymann D, Redini F (2005) Zoledronic acid suppresses lung metastasis and prolongs overall survival of osteosarcoma-bearing mice. Cancer 104:2522-2529

Ozger H, Eralp L, Atalar AC, Toker B, Esberk Ates L, Sungur M, Bilgic B, Ayan I (2009) The effect of resistance-related proteins on the prognosis and survival of patients with osteosarcoma: an immunohistochemical analysis. Acta Orthop Traumatol Turc 43:28-34

Porter JR, Fritz CC, Depew KM (2010) Discovery and development of Hsp90 inhibitors: a promising pathway for cancer therapy. Curr Opin Chim Biol 14:412-420

Qian L, Zhang Z, Shi M, Yu M, Hu M, Xia Q, Shen B, Guo N (2006) Expression and distribution of Hsp27 in response to G418 in different human breast cancer cell lines. Histochem Cell Biol 126:593-601

Romanucci M, Bongiovanni L, Marruchella G, Marà M, Di Guardo G, Preziosi R, Della Salda L (2005) Heat shock proteins expression in canine intracutaneous cornifying epithelioma and squamous cell carcinoma. Vet Dermatol 16:108-116

Romanucci M, Marinelli A, Sarli G, Della Salda L (2006) Heat shock protein expression in canine malignant mammary tumours. BMC Cancer 6:171

Slayter MV, Boosinger TR, Pool RR, Dämmrich K, Misdorp W, Larsen S (1994) Histological classification of bone and joint tumors of domestic animals. In: 2nd series Volume I, World Health Organization AFIP, Washington, DC

Straw RC, Withrow SJ, Richter SL, Powers BE, Klein MK, Postorino NC, LaRue SM, Ogilvie GK, Vail DM, Morrison WB, McGee M, Dickinson K (1991) Amputation and cisplatin for treatment of canine osteosarcoma. J Vet Intern Med 5:205-210

Trepel J, Mollapour M, Giaccone G, Neckers L (2010) Targeting the dynamic Hsp90 complex in cancer. Nat Rev Cancer 10:537-549 
Trieb K, Lechleitner T, Lang S, Windhager R, Kotz R, Dirnhofer S (1998) Heat shock protein 72 expression in osteosarcoma correlates with good response to neoadjuvant chemotherapy. Human Pathol 29:1050-1055

Trieb K, Lang S, Kotz R (2000) Heat-shock protein72 in human osteosarcoma: T-lymphocyte reactivity and cytotoxicity. Pediatr Haematol Oncol 17:355-364
Uozaki H, Ishida T, Kakiuchi C, Horiuchi H, Gotoh T, Iijima T, Imamura T, Machinami R (2000) Expression of heat shock proteins in osteosarcoma and its relationship to prognosis. Pathol Res Pract 196:665-673

Yamamoto K, Okamoto A, Isonishi S, Ochiai K, Ohtake Y (2001) Heat shock protein 27 was up-regulated in cisplatin resistant human ovarian tumor cell line and associated with the cisplatin resistance. Cancer Lett 168:173-181 